\title{
A New Multi-Component Diffuse Interface Model with Peng-Robinson Equation of State and its Scalar Auxiliary Variable (SAV) Approach
}

\author{
Zhonghua Qiao ${ }^{1, *}$, Shuyu Sun ${ }^{2,3}$, Tao Zhang ${ }^{2,3}$ and Yuze Zhang ${ }^{1}$ \\ ${ }^{1}$ Department of Applied Mathematics, The Hong Kong Polytechnic University, \\ Hung Hom, Hong Kong. \\ 2 Computational Transport Phenomena Laboratory (CTPL), Division of Physical \\ Sciences and Engineering (PSE), King Abdullah University of Science and \\ Technology (KAUST), Thuwal 23955-6900, Kingdom of Saudi Arabia. \\ ${ }^{3}$ Institute of Geophysics and Geomatics, China University of Geosciences, \\ Wuhan 430074, P.R. China.
}

Received 8 February 2019; Accepted (in revised version) 11 April 2019

\begin{abstract}
A new multi-component diffuse interface model with the Peng-Robinson equation of state is developed. Initial values of mixtures are given through the NVT flash calculation. This model is physically consistent with constant diffusion parameters, which allows us to use fast solvers in the numerical simulation. In this paper, we employ the scalar auxiliary variable (SAV) approach to design numerical schemes. It reformulates the proposed model into a decoupled linear system with constant coefficients that can be solved fast by using fast Fourier transform. Energy stability is obtained in the sense that the modified discrete energy is non-increasing in time. The calculated interface tension agrees well with laboratory experimental data.
\end{abstract}

AMS subject classifications: 65N06, 65B99

Key words: Peng-Robinson equation of state, multi-component diffuse interface model, scalar auxiliary variable approach, energy stable scheme.

\section{Introduction}

In reservoir engineering and chemical flows, multi-component and multi-phase fluid systems are unneglectable with important roles in the thorough and accurate understanding of flow behaviors in a large range of applications $[2-4,6,15,17,22]$. In numerical

*Corresponding author. Email addresses: zqiao@polyu.edu.hk (Z. Qiao), shuyu. sun@kaust.edu.sa (S. Sun), tao.zhang.1@kaust.edu.sa (T. Zhang), 16903152r@connect.polyu.hk (Y. Zhang) 
simulations, it is a key effort to determine whether the studied fluid mixtures remain in one single phase or split into multiple phases including oil phase, water phase, gas phase, etc. Thermodynamic equilibrium conditions are the basic rule to control physical properties of the mixture fluid flow, such as composition and density of each phase if split, and whether the phase split occurs at all. Recently, realistic equations of state (EOS) (e.g. Peng-Robinson EOS [18]) have attracted more and more attention in the multi-component multi-phase flow simulation, which is very useful in the study of thermodynamic mechanism. It can be applied in many areas, especially in the pore scale modeling of subsurface fluid flow $[7-9,11]$. The main cause of capillarity, a major immiscible two-phase flow mechanism for systems with a strong wettability preference, is often attributed to the interface tension, which is mainly determined by phase behaviors of multi-component fluids. In order to capture the phase properties and behaviors better, diffuse interface models based on Peng-Robinson EOS have been widely studied in recent years [6,11].

While modeling the multi-component system, the mobility tensor is a significant element to be considered. Mobility, which is a variable defined in diffuse interface models, plays an essential role to keep the developed model consistent with thermodynamic laws. The mobility matrix $M$ shall be symmetric and positive semi-definite so that Onsager's reciprocal principle and the second law of thermodynamics are satisfied. Different methods have been proposed to model the mobility tensor, which could be summarized into three types. The first one is to define mobility as a diagonal matrix with positive diagonal elements, which satisfies the aforementioned two principles and is convenient to implement. Only the diffusivity of each component is considered, so that the mobility tensor could be represented simply as: $M_{i i}$. The second one is to take mobility matrix as a full matrix, and namely we have two tensors $M_{i i}$ and $M_{i j}$. It should be noted that the mole mean diffusivity matrix $D_{i i}=0$ but $D_{i j}>0$. The third one is to use mass mean diffusivity instead of mole mean diffusivity. More details on the modeling of the mobility tensor could be found in [13] and references therein. In this paper, inspiring from the first choice of mobility, we propose a new multi-component two phase diffuse interface model in order to use certain fast calculation approaches.

How to select the initial value of multi-component mixture becomes an important modeling issue when the realistic equation of state (EOS) is involved. To handle this, we need to consider a phase splitting problem. The NPT flash calculation (temperature T, pressure $\mathrm{P}$ and composition $\mathrm{N}$ ) and the NVT flash calculation (temperature $\mathrm{T}$, volume $\mathrm{V}$ and composition N) are two common phase splitting approaches. As discussed in [21], classical coupled schemes based on the NPT flash calculation suffer from a few essential limitations, such as the requirement of constructing a pressure equation as there is no intrinsic pressure equation. An alternative modeling framework, based on the NVT flash calculation with moles, volume and temperature as the primal state variables, has been actively studied very recently $[7,8]$. It allows us to get the molar density of gas and liquid of a specific substance when the phase transition occurs, and is better posed than the NPT flash calculation. The obtained solution can be used as the initial value of the 
diffuse interface model with given substances.

Efficient numerical schemes are highly needed for solving such diffuse interface models with Peng-Robinson EOS. The main challenge is the strong non-linearity in the function of bulk Helmholtz free energy density, which usually becomes more serious as the number of components increases. Several approaches have been carried out to construct energy stable schemes for diffuse interface models with Peng-Robinson EOS. In $[6,11,12,19,20,22]$, energy stable schemes are proposed by using the convex-splitting strategy, which is a popular used approach for solving diffuse interface problems. The modified Newton's method with a relaxation parameter, which is mentioned in [9], is another approach to guarantee the energy decay property while constructing the schemes. In addition, a classical fully implicit scheme is studied in [10] to deal with a multicomponent system with Peng-Robinson EOS. Recently, the invariant energy quadratization (IEQ) scheme, is also used to study the Peng-Robinson EOS problems [14]. In this paper, we use the scalar auxiliary variable (SAV) approach $[24,25]$ to design a second order SAV Crank-Nicolson (SAV-CN) scheme. It leads to an unconditionally energy stable numerical scheme of decoupled linear equations with constant coefficients that can be solved fast by using fast Fourier transform.

The rest of the paper is organized as follows. In Section 2, we will develop a multicomponent two-phase flow model with the mobility tensor involved. The method of initial value selection, which is based on the NVT framework, is also given in this section. In Section 3, we will propose a second order SAV scheme for this model. The energy stability is also studied in this section. In Section 4, several numerical tests are designed and implemented to demonstrate the effectiveness of the proposed model and the numerical scheme. Some concluding remarks are given in Section 5.

\section{Mathematical modelling of multi-component two-phase flow}

\subsection{Governing equations}

We now formulate a thermodynamic consistent mathematical model to describe multicomponent two-phase flow based on the Peng-Robinson EOS, which is widely used in petroleum industry. It can accurately represent the thermodynamic properties of hydrocarbon mixtures in the multi-phase fluid flow. We model the flow under an isothermal condition, i.e, with a constant temperature $T$.

Governing equations can be written as the following form

$$
\begin{aligned}
& \frac{\partial n_{i}}{\partial t}+\nabla \cdot J_{i}=0, \\
& J_{i}=-\sum_{j=1}^{m} M_{i j} \nabla \mu_{j},
\end{aligned}
$$

for $i=1,2, \cdots, m$. In this paper, we consider periodic boundary conditions. Here $n_{i}, J_{i}$ and $\mu_{i}$ are the molar density, the diffusive flux and the total chemical potential of the $i$-th 
component, respectively. $M=\left(M_{i j}\right)_{m \times m}$ is the mobility tensor, which should be symmetric and at least positive semi-definite (in most cases, strictly positive definite) to satisfy Onsager's reciprocal principle and the second law of thermodynamics. In [13], Kou and Sun introduced several approaches to form the mobility term. In this paper, we give a new approach of $M$ by using a diagonal matrix with positive diagonal elements to meet the above requirements. We set

$$
M_{i i}=\frac{D_{i} \frac{N_{i}^{0}}{|\Omega|}}{R T}, \quad i=1,2, \cdots, m,
$$

where $N_{i}^{0}$ is the total particle amount of the $i$-th component at the initial state and $|\Omega|$ is the calculated volume (area in 2D). $R$ stands for the universal gas constant and $D_{i}>0$ is the diffusion coefficient of component $i$. Therefore, the diffusion flux can be written as

$$
J_{i}=-\frac{D_{i} \frac{N_{i}^{0}}{R T}}{R T} \nabla \mu_{i}, \quad i=1,2, \cdots m .
$$

We need to mention that the above modeling of mobility by a diagonal matrix allows us to design certain fast calculation numerical schemes (such the SAV scheme studied in this paper) because of its constant coefficients.

Using the mobility (2.3), the origin problem can be rewritten as

$$
\begin{gathered}
\frac{\partial n_{i}}{\partial t}+\nabla \cdot J_{i}=0, \\
J_{i}=-M_{i i} \nabla \mu_{i}
\end{gathered}
$$

for $i=1,2, \cdots, m$. The total chemical potential of the $i$-th component $\mu_{i}$ as used in (2.1) has the bulk contribution $\mu_{b, i}$ and the gradient contribution $\mu_{\Delta, i}$.

$$
\mu_{i}=\mu_{b, i}-\mu_{\Delta, i}=\mu_{b, i}-\sum_{j=1}^{m} c_{i j} \Delta n_{j}, \quad i=1,2, \cdots m,
$$

where the influence parameter $c_{i j}$ is usually assumed to be a constant and its expression can be seen in the appendix. The bulk part $\mu_{b, i}$ is the derivative of the bulk Helmholtz free energy density $f_{b}$ with respect to $n_{i}$. The expression of $f_{b}$ of the Peng-Robinson EOS case can also be found in the appendix. The total energy of the system is defined as

$$
F=F_{b}+F_{\nabla}
$$

where

$$
\begin{aligned}
& F_{b}=\int_{\Omega} f_{b}(n) d x \\
& F_{\nabla}=\frac{1}{2} \int_{\Omega} \sum_{i}^{m} \sum_{j}^{m} c_{i j} \nabla n_{i} \cdot \nabla n_{j} d x .
\end{aligned}
$$




\subsection{Initial value calculation for the multi-component dynamic system}

For the multi-component multi-phase flow simulation based on realistic equations of state, a tricky issue challenging a reliable numerical result is how to choose applicable initial values on the molar density distribution of the components. To address this issue, in this paper, we provide a brief introduction on how to conduct a reasonable initial value calculation based on the thermodynamic theory of flash calculation [16].

In two-phase compositional flow, compositions of chemical components in each phase change with thermodynamic conditions, and that is what flash calculations determine. Phase splitting may occur or disappear with the variation of temperature or pressure, which adds on the difficulty of the composition determination. Wide additional properties at phase equilibrium statements can also been predicted through flash calculation. A general phase splitting problem is often defined with an assumption of a certain given thermodynamic equilibrium state among the components. Common phase splitting formulations include the NPT flash (with constant temperature $T$, pressure $P$ and composition $N$ ) and the NVT flash (with constant temperature $T$, volume $V$ and composition $N)$.

Here, we want to introduce the NVT flash calculation which is a relatively new approach to deal with the phase splitting problem. First of all, we specify the overall composition, i.e. mole fraction of each species in the overall fluid mixture consisting $\mathrm{n}$ components and possibly multiple phases and we define $z_{i}=\frac{N_{i}}{N}$ as the overall mole fraction of the $i$-th component in the entire mixture. $N_{i}$ is the total amount of $i$-th component. $N=\sum_{i}^{m} N_{i}$ and $V$ is the total amount and total volume respectively. Then we will go through two steps to get the molar concentrations of the substances that can guarantee the phase separation numerically and the given mixture is not thermodynamic phasestable.

At the beginning, we need to give some notations: $c=\frac{N}{V}$ is the total molar density of the mixture and $c z_{i}=\frac{N_{i}}{V}, i=1, \cdots, m$ is the molar concentration of $i$-th component. $c^{\prime}$ is the trial molar density or the total molar density of the new phase (which can be found in Fig. 1, and details will be discussed shortly). $c^{\prime \prime}$ is the molar density of the original phase after the phase separation (which can be found in Fig. 2, and details will be discussed shortly). $V_{i}^{\prime}$ is the volume of $i$-th component in the new phase and $V_{i}^{\prime \prime}$ is the volume of $i$ th component in the original phase after the phase separation. $V^{\prime}=\sum_{i} V_{i}^{\prime}$ and $V^{\prime \prime}=\sum_{i} V_{i}^{\prime \prime}$. $N_{i}^{\prime}$ is the particle number of $i$-th component in the new phase and $N_{i}^{\prime \prime}$ is the particle number of $i$-th component in the original phase after the phase separation. $N^{\prime}=\sum_{i} N_{i}^{\prime}$ and $N^{\prime \prime}=\sum_{i} N_{i}^{\prime \prime}$ and we denote $\bar{y}_{i}=\frac{N_{i}^{\prime \prime}}{N^{\prime \prime}}, i=1, \cdots, m$.

\section{STEP 1. Infinitesimal splitting}

It is believed that, if the phase splitting happens, the new phase (here we call it the trial phase) will form with a infinitesimal volume (here we use $0^{+}$to describe this volume) at the very beginning of the phase separation. So our first step is to determine whether or not this phenomenon happens under given conditions. 


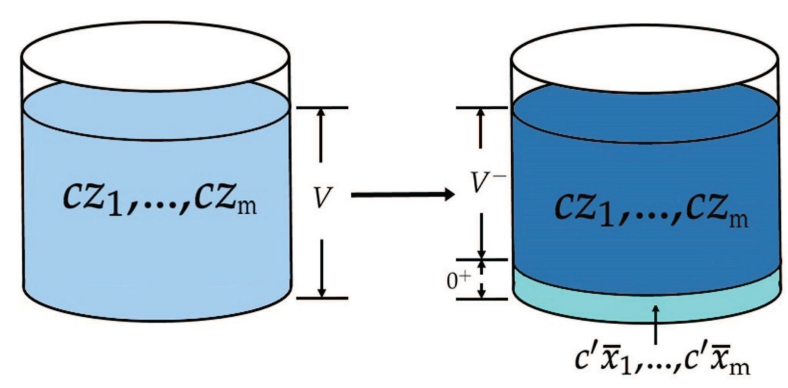

Figure 1: Infinite small splitting.

As we can see in the Fig. 1, if the phenomenon happens, the original single-phase mixture (with the $m$ components' molar densities $c z_{1}, \cdots, c z_{m}$ ) will split into the trial phase part (infinitesimal volume part) with the volume $0^{+}$and the rest part with the volume $V^{-}=V-0^{+}$during the progress of the infinite small splitting. In this step, our aim is to find the trial molar density $c^{\prime}$ of the new phase.

First, we need to calculate the saturation pressure $p^{\text {sat }}$ which has the following form

$$
p^{\text {sat }}=p_{c} \exp \left[5.37(1+\omega)\left(1-\frac{T_{c}}{T}\right)\right],
$$

where

$$
\omega=\frac{3}{7}\left(\frac{\log _{10}\left(\frac{p_{c}}{p_{\mathrm{atm}}}\right)}{\frac{T_{c}}{T_{b}}-1}\right) .
$$

Given critical properties $T_{c}, P_{\mathcal{c}}$ and boiling point $T_{P}$ of different components, we will get different $p^{\text {sat }}$ of different components. Here we note that the $p_{\text {atm }}$ represents the unit standard atmosphere pressure. Usually it has the following relation with the Mpa: $1 \mathrm{Mpa} \approx 9.8 \mathrm{~atm}$. After we have saturation pressure $p_{i}^{\text {sat }}$ for each component, we will calculate the trial phase composition under the framework of the NVT flash calculation.

The truth is, from the beginning, we do not know the phase state of the mixture. So we have following two assumptions.

- Case 1: If the trial phase is in the liquid phase we first calculate the total initial pressure by

$$
p_{\text {ini }}=\sum_{i}^{m} p_{i}^{\text {sat }}(T) z_{i}
$$

then we give the formulation of the trial phase composition (liquid-like)

$$
\bar{x}_{i}=\frac{p_{i}^{\text {sat }}}{p_{\text {ini }}} z_{i}, \quad i=1, \cdots, m .
$$

Then we calculate the compressibility factor $Z$ of the system by solve the following equation based on the Peng-Robinson EOS (Different equation of state has different 
formulation which respects to $Z$ ).

$$
Z^{3}-(1-B) Z^{2}+\left(A-2 B-3 B^{2}\right) Z-\left(A B-B^{2}-B^{3}\right)=0,
$$

where

$$
\begin{aligned}
& A=\frac{a\left(T_{c}\right) p_{\text {ini }}}{R^{2} T^{2}}, \\
& B=\frac{b p_{\text {ini }}}{R T} .
\end{aligned}
$$

Here the expression of $a\left(T_{c}\right)$ and $b$ can be found in appendix. We use all real solutions of the equation (2.11) to do the following analysis.

- Case 2: If the trial phase is in the gas phase, first we calculate the trial phase composition (gas-like)

$$
\bar{x}_{i}=\frac{\frac{z_{i}}{p_{i}^{\text {sat }}}}{\sum \frac{z_{j}}{p_{j}^{\text {sat }}}}, \quad i=1, \cdots, m .
$$

Then the initial pressure is given by

$$
p_{\text {ini }}=\Sigma p_{i}^{\text {sat }}(T) \bar{x}_{i}
$$

We can get $Z$ in the same way as in Case 1 .

We can use the obtained $Z$ to calculate the total molar density of trail phase $c^{\prime}$ by the following relation (the non-ideal gas equation)

$$
p_{\text {ini }}=Z c^{\prime} R T \text {. }
$$

Here, we need to mention that $p_{\text {ini }}$ we used in the (2.15) should be calculated under the same assumption with the $Z$ we choose.

Then we will show a special method to determine whether or not the system will experience a phase separation under the overall composition that we gave at the beginning. Here we introduce the tangent plane distance function D

$$
\begin{aligned}
D\left(T, 1, c^{\prime} \bar{x}_{1}, \cdots, c^{\prime} \bar{x}_{m}\right)= & \Sigma\left[\mu_{i}\left(\left(T, 1, c^{\prime} \bar{x}_{1}, \cdots, c^{\prime} \bar{x}_{m}\right)\right)-\mu_{i}\left(\left(T, 1, c z_{1}, \cdots, c z_{m}\right)\right)\right] c^{\prime} \bar{x}_{i} \\
& -\left[p\left(\left(T, 1, c^{\prime} \bar{x}_{1}, \cdots, c^{\prime} \bar{x}_{m}\right)\right)-p\left(\left(T, 1, c z_{1}, \cdots, c z_{m}\right)\right)\right] .
\end{aligned}
$$

The function $D$ is used to determine whether the phase separation will happen. If $D$ is less than 0 , it means there will have a phase separation and the molar density of trial phase is $c^{\prime}$. Otherwise we need to use another $Z$ to do the same process (more details can be seen in the paper by Jiri [16]). If we fail to find the appropriate $c^{\prime}$ from all the $Z$ we got in Case 1 and Case 2, we need to consider a new set of $z_{i}$ for the purpose of two-phase system specification. 


\section{STEP 2. Finite-amount splitting}

The existing of $c^{\prime}$ means the original single-phase mixture will experience the phase separation with the initial fraction $z_{i}$. Under the assumption that the molar density of the new phase still equals to the trial phase molar density $c^{\prime}$ and the phase composition still equal to $\bar{x}_{i}$ (which means $\bar{x}_{i}=\frac{N_{i}^{\prime}}{N^{\prime}}$ ) after the phase separation. We can get molar density $c^{\prime \prime}$, the phase composition $\bar{y}_{i}$ and the total volume $V^{\prime \prime}$ of another phase. We call this progress the "finite-amount splitting" and it can be shown in the Fig. 2.

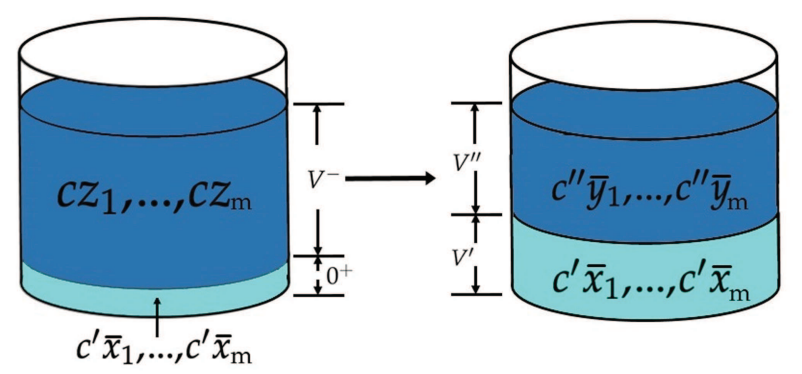

Figure 2: Finite-amount splitting.

To be specific, in this step, we calculate the molar density of both phases of the $i$-th component by using

$$
\begin{aligned}
& V^{\prime}+V^{\prime \prime}=V, \\
& c^{\prime} V^{\prime}+c^{\prime \prime} V^{\prime \prime}=c V, \\
& f_{b}\left(c^{\prime}\right) V^{\prime}+f_{b}\left(c^{\prime \prime}\right) V^{\prime \prime}-f_{b} V<0,
\end{aligned}
$$

where $f_{b}$ represents the Helmholtz free energy density. Here we use the bisection method to get the value $V^{\prime \prime}$ and $c^{\prime \prime}$, first we set $V^{\prime}=0.5 V$ and we can get the $c^{\prime \prime}$ and $V^{\prime \prime}$ by the relation

$$
\begin{aligned}
& V^{\prime \prime}=V-V^{\prime}, \\
& c^{\prime \prime}=\frac{c V-c^{\prime} V^{\prime}}{V^{\prime \prime}},
\end{aligned}
$$

where $V$ is the initial volume which we set arbitrary, for simplicity, we set $V=1$. Then we put the $c^{\prime}, V^{\prime}, c^{\prime \prime}, V^{\prime \prime}$ into the determination equation $f_{b}\left(c^{\prime}\right) V^{\prime}+f_{b}\left(c^{\prime \prime}\right) V^{\prime \prime}-f_{b} V<0$. If the inequality is established, we can get the molar densities of two phases under the given thermodynamic properties.

For the multi-component systems, we can further calculate the composition of both phases by using the relation

$$
c^{\prime} \bar{x}_{i} V^{\prime}+c^{\prime \prime} \bar{y}_{i} V^{\prime \prime}=c z_{i} V, \quad i=1, \cdots, m,
$$

which leads to

$$
\bar{y}_{i}=\frac{c z_{i} V-c^{\prime} \bar{x}_{i} V^{\prime}}{c^{\prime \prime} V^{\prime \prime}}, \quad i=1, \cdots, m
$$




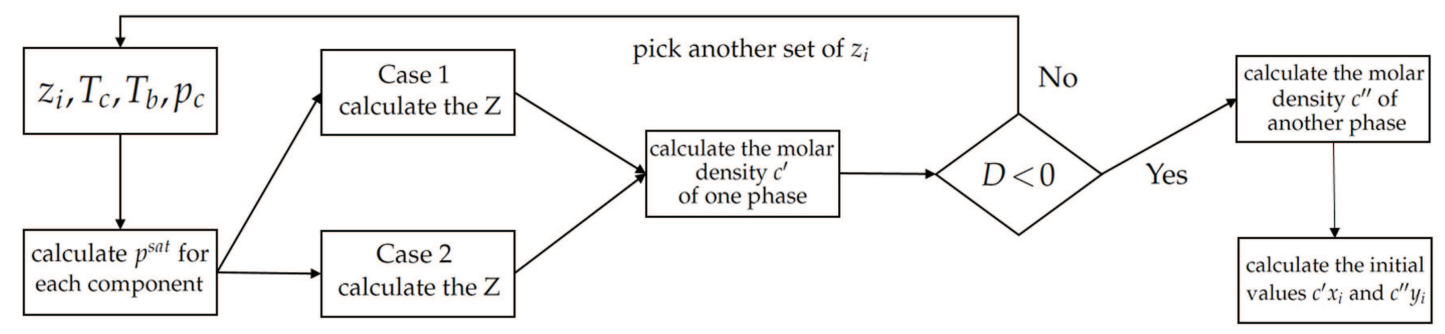

Figure 3: Flowchart of the NVT flash calculation.

Similarly, we can calculate $\bar{y}_{i}$ by using

$$
f_{b}\left(c^{\prime} \bar{x}_{1}, \cdots, c^{\prime} \bar{x}_{m}\right) V^{\prime}+f_{b}\left(c^{\prime \prime} \bar{y}_{1}, \cdots, c^{\prime \prime} \bar{y}_{m}\right) V^{\prime \prime}-f_{b}\left(c z_{1}, \cdots, c z_{m}\right) V<0
$$

as the determination function. Until now, we can get the molar concentrations $c^{\prime} \bar{x}_{i}$ and $c^{\prime \prime} \bar{y}_{i}$ of each component of different phases. We can use these molar concentrations as initial values of our model, or we can optimize them further using additional schemes, e.g. the method in [16].

Fig. 3 gives a flowchart of the NVT flash calculation. Based on the above flash calculation scheme, molar density distribution of both the two components can be determined as the initial condition of our further numerical simulation. For the convenience of researchers in this field, we pre-computed two-phase two-component system with the procedure described method and the composition results can be found in Table 1.

Table 1: Initial values $\left(\mathrm{mol} / \mathrm{m}^{3}\right)$ for methane $\left(n_{1}\right)$ and $n$-decane $\left(n_{2}\right)$ in gas and liquid phases.

\begin{tabular}{||c|c|c|c|c||}
\hline Temperature $(K)$ & $n_{1}$ (liquid) & $n_{1}$ (gas) & $n_{2}$ (liquid) & $n_{2}$ (gas) \\
\hline 450 & 4062 & 1028 & 438 & 3522 \\
\hline 400 & 3832 & 1428 & 488 & 2938 \\
\hline 350 & 3675 & 1536 & 512 & 2861 \\
\hline 300 & 3569 & 1648 & 564 & 2497 \\
\hline
\end{tabular}

\section{SAV approach of the multi-component model with Peng-Robinson EOS}

\subsection{Model reformulation}

Considering the multi-component two-phase model (2.4), we use the SAV approach to reformulate it. First, we introduce $\mathbb{V}(t)$, which has the following form

$$
\mathbb{V}(t)=\sqrt{F_{b}+\sum_{i=1}^{m} C_{T, i} N_{i}^{t}} .
$$


Here, $C_{T, i} \geq 0$ is the thermodynamic coefficient of component $i$ to ensure $F_{b}+\sum_{i=1}^{m} C_{T, i} N_{i}^{t} \geq$ 0 , and we need to choose the $C_{T, i}$ based on the temperature $T$ but independent of the molar density. One thing we would like to mention here is that $F_{b}$ is always larger than 0 during all the numerical experiments we carried out for real substances. Using $\mathbb{V}(t)$ to rewrite the chemical potential, we can get

$$
\mu_{i}=\frac{\mathbb{V}(t)}{\sqrt{F_{b}+\sum_{i=1}^{m} C_{T, i} N_{i}^{t}}} \mu_{b, i}-\sum_{j=1}^{m} c_{i j} \Delta n_{j} .
$$

With this, the origin system (2.4) changes to for $i=1,2, \cdots, m$

$$
\begin{aligned}
& \frac{\partial n_{i}}{\partial t}=M_{i i} \Delta \mu_{i}, \\
& \mu_{i}=\frac{\mathbb{V}(t)}{\sqrt{F_{b}+\sum_{i=1}^{m} C_{T, i} N_{i}^{t}}} \mu_{b, i}-\sum_{j=1}^{m} c_{i j} \Delta n_{j}, \\
& \frac{\partial \mathbb{V}}{\partial t}=\sum_{i=1}^{m} \int_{\Omega} \frac{\mu_{b, i}}{2 \sqrt{F_{b}+\sum_{j=1}^{m} C_{T, j} N_{j}^{t}}} \frac{\partial n_{i}}{\partial t} d x .
\end{aligned}
$$

The total energy $F$ can be reformulated as $F=F_{\nabla}+\mathbb{V}^{2}$. The following energy law can be easily obtained by integrating the three equations in (3.2) with $\mu_{i}, \frac{\partial n_{i}}{\partial t}$ and $\mathbb{V}$, respectively and using periodic boundary conditions.

Lemma 3.1. The total energy F satisfies the following energy law:

$$
\frac{d F}{d t}=-\sum_{i=1}^{m} M_{i i} \int_{\Omega} \nabla \mu_{i} \nabla \mu_{i} d x
$$

Remark 3.1. (3.4) implies that the energy $F$ is non-increasing in time. It is easy to get that (3.2) also satisfies the mass conservation

$$
\frac{d}{d t} \int_{\Omega} n_{i} d x=0, \quad i=1,2, \cdots, m .
$$

\subsection{Notations of spectral discretization}

In this section, we will give notations of some spatial operators for the spectral collocation method on the two-dimensional space $\Omega=(0, X) \times(0, Y)$.

Let $N_{x}, N_{y}$ be any positive even numbers, and the $N_{x} \times N_{y}$ mesh $\Omega_{h}$ of $\Omega$ can be described as the following nodes set $\left(x_{i}, y_{j}\right)$, where $x_{i}=i h_{x}, y_{j}=j h_{y}, 1 \leq i \leq N_{x}, 1 \leq j \leq N_{y}$. $h_{x}=\frac{X}{N_{x}}, h_{y}=\frac{Y}{N_{y}}$. We define index sets

$$
\begin{aligned}
& J_{h}=\left\{(i, j) \in \mathbb{N}^{2} \mid 1 \leq i \leq N_{x}, 1 \leq j \leq N_{y}, 1 \leq k\right\}, \\
& \hat{J}_{h}=\left\{(l, m) \in \mathbb{Z}^{2} \mid-\frac{N_{x}}{2}+1 \leq l \leq \frac{N_{x}}{2},-\frac{N_{y}}{2}+1 \leq m \leq \frac{N_{y}}{2}\right\} .
\end{aligned}
$$


Then we define all the periodic grid functions on $\Omega_{h}$ as $F_{h}$, which has the following form

$$
F_{h}=\left\{f: \Omega_{h} \rightarrow R \mid f_{i+l N_{x}, j+m N_{y}}=f_{i, j}, \text { for any }(i, j) \in J_{h} \text { and }(l, m) \in \mathbb{Z}^{2}\right\} .
$$

For any function $f \in F_{h}$, we can define the following 2-D Fourier transform $\hat{f}=P f$ and the inverse Fourier transform $f=P^{-1} \hat{f}$ by

$$
\begin{aligned}
& \hat{f}_{l, m}=\frac{1}{N_{x} N_{y}} \sum_{(i, j) \in J_{h}} f_{i, j} \exp \left(-i \frac{2 l \pi}{X} x_{i}\right) \exp \left(-i \frac{2 m \pi}{Y} y_{j}\right), \quad(l, m) \in \hat{J}_{h} ; \\
& f_{i, j}=\sum_{(l, m) \in \hat{J}_{h}} \hat{f}_{l, m} \exp \left(i \frac{2 l \pi}{X} x_{i}\right) \exp \left(i \frac{2 m \pi}{Y} y_{j}\right), \quad(i, j) \in J_{h} .
\end{aligned}
$$

Let $\hat{F}_{h}=\left\{P f \mid f \in F_{h}\right\}$. First order partial operators $\hat{D}_{x}, \hat{D}_{y}$ and $\hat{D}_{z}$ are defined on $\hat{F}_{h}$ as follows:

$$
\left(\hat{D}_{x} \hat{f}\right)_{l, m}=\left(\frac{2 l \pi i}{X}\right) \hat{f}_{l, m}, \quad\left(\hat{D}_{y} \hat{f}\right)_{l, m}=\left(\frac{2 m \pi i}{Y}\right) \hat{f}_{l, m}, \quad(l, m) \in \hat{J}_{h} .
$$

Then the spectral form of second order partial operators can be written as

$$
D_{x}^{2}=P^{-1} \hat{D}_{x}^{2} P, \quad D_{y}^{2}=P^{-1} \hat{D}_{y}^{2} P .
$$

We can define the discrete Laplace operator $\Delta_{h}$ as

$$
\Delta_{h} f=D_{x}^{2} f+D_{y}^{2} f .
$$

The inner product can also be denoted as

$$
(f, g)_{h}=h_{x}^{2} h_{y}^{2} \sum_{i=1}^{N_{x}} \sum_{j=1}^{N_{y}} f_{i, j} \cdot g_{i, j} .
$$

\subsection{A SAV-CN scheme of a two-component model with Peng-Robinson EOS}

In this paper, we consider a two-component model with Peng-Robinson EOS. We solve the system in the time interval $[0, \mathbf{T}]$ and the space domain $\Omega=[0, X]^{2}$. For a given positive integer $N_{t}$, we set the time step $\Delta t$ as $\Delta t=\frac{\mathrm{T}}{N_{t}}$. For a given positive integer $N_{s}$, we set the gride size $h$ as $h=\frac{X}{N_{s}}$. The SAV-CN scheme of the system (2.4) is constructed by using the spectral collocation method in space as follows: for $0 \leq s \leq N_{t}-1$, find $n^{s+1} \in F_{h}$ such that

$$
\begin{aligned}
& \frac{n_{i}^{s+1}-n_{i}^{s}}{\Delta t}=M_{i i} \Delta_{h} \mu_{i}^{s+1 / 2}, \\
& \mu_{i}^{s+1 / 2}=-\frac{1}{2} \sum_{j=1}^{2} c_{i j}\left(\Delta_{h} n_{j}^{s+1}+\Delta_{h} n_{j}^{s}\right)+\frac{\mu_{b, i}^{s+1 / 2}}{2 \mathbb{F}^{1 / 2}}\left(\mathbb{V}^{s+1}+\mathbb{V}^{s}\right), \quad i=1,2, \\
& \mathbb{V}^{s+1}-\mathbb{V}^{s}=\sum_{j=1}^{2}\left(\frac{\mu_{b, j}^{s+1 / 2}}{2 \mathbb{F}^{s+1 / 2}}, n_{j}^{s+1}-n_{j}^{s}\right)_{h},
\end{aligned}
$$


where $\mathbb{F}^{s+1 / 2}=\sqrt{F_{b}\left(\hat{n}_{1}^{s+1 / 2}, \hat{n}_{2}^{s+1 / 2}\right)+\sum_{i=1}^{2} C_{T, i} N_{i}^{t}}$ and $\hat{n}_{i}{ }^{s+1 / 2}$ can be regarded as an explicit approximation of $n_{i}^{s+1 / 2}$. However, due to the high nonlinearity of the Helmholtz free energy from Peng-Robinson EOS, it has a strict constrain on the time step $\Delta t$ using the explicit approximation. So we employ the following equation for solving $\hat{n}_{i}^{s+1 / 2}$

$$
\frac{\hat{n}_{i}^{s+1 / 2}-n^{s}}{(1 / 2) \Delta t}=M_{i} \Delta_{h} \mu_{b, i}^{s}-\sum_{j=1}^{2} c_{i j} \Delta_{h} \hat{n}_{j}^{s+1 / 2}, \quad i=1,2 .
$$

Due to $c_{i j}=c_{j i}$, we can have the following property

$$
\left(\sum_{j=1}^{2} c_{i j} \Delta_{h}\left(n_{j}^{s+1}+n_{j}^{s}\right), n_{i}^{s+1}-n_{i}^{s}\right)_{h}=\sum_{j=1}^{2} c_{i j}\left[\left(\Delta_{h} n_{j}^{s+1}, n_{i}^{s+1}\right)_{h}-\left(\Delta_{h} n_{j}^{s}, n_{i}^{s}\right)_{h}\right], \quad i=1,2 .
$$

Using this property, we multiply three equations of (3.5) with $\Delta t \mu^{s+1 / 2}, n_{i}^{s+1 / 2}-n_{i}^{s}$ and $\mathbb{V}^{s+1}+\mathbb{V}^{s}$, respectively, and then get for $i=1,2$

$$
\begin{aligned}
\left(\mu_{i}^{s+1 / 2}, n_{i}^{s+1}-n_{i}^{s}\right)_{h}= & \left(\Delta t \mu_{i}^{s+1 / 2}, M_{i i} \Delta_{h} \mu_{i}^{s+1 / 2}\right)_{h}, \\
\left(\mu_{i}^{s+1 / 2}, n_{i}^{s+1}-n_{i}^{s}\right)_{h}= & -\frac{1}{2}\left(\sum_{j=1}^{2} c_{i j}\left(\Delta_{h} n_{j}^{s+1}+\Delta_{h} n_{j}^{s}\right), n_{i}^{s+1}-n_{i}^{s}\right)_{h} \\
& +\left(\frac{\mu_{b, i}^{s+1 / 2}}{2 \mathbb{F}^{1 / 2}}\left(\mathbb{V}^{s+1}+\mathbb{V}^{s}\right), n_{i}^{s+1}-n_{i}^{s}\right)_{h} \\
\left(\mathbb{V}^{s+1}\right)^{2}-\left(\mathbb{V}^{s}\right)^{2}= & \sum_{j=1}^{2}\left(\frac{\mu_{b, i}^{s+1 / 2}}{2 \mathbb{F}^{s+1 / 2}}\left(\mathbb{V}^{s+1}+\mathbb{V}^{s}\right), n_{i}^{s+1}-n_{i}^{s}\right)_{h} .
\end{aligned}
$$

Then

$\sum_{i=1}^{2}\left(\Delta t \mu_{i}^{s+1 / 2}, M_{i i} \Delta_{h} \mu_{i}^{s+1 / 2}\right)_{h}=-\frac{1}{2} \sum_{i=1}^{2} \sum_{j=1}^{2} c_{i j}\left[\left(\Delta_{h} n_{j}^{s+1}, n_{i}^{s+1}\right)_{h}-\left(\Delta_{h} n_{j}^{s}, n_{i}^{s}\right)_{h}\right]+\left(\mathbb{V}^{s+1}\right)^{2}-\left(\mathbb{V}^{s}\right)^{2}$.

In the mean time, we define the modified discrete total energy at time level $s$ as

$$
F_{m}^{s}=-\frac{1}{2} \sum_{i=1}^{2} \sum_{j=1}^{2} c_{i j}\left(\Delta_{h} n_{j}^{s}, n_{i}^{s}\right)_{h}+\left(\mathbb{V}^{s}\right)^{2},
$$

and the operator $\Delta_{h}$ is negative definite. It is now clear that the following theorem holds:

Theorem 3.1. The second order $S A V-C N$ scheme (3.5) is unconditional energy stable meaning that for any $\Delta t>0$ we have

$$
F_{m}^{s+1}-F_{m}^{s} \leq 0 .
$$

Remark 3.2. The following mass conservation property of (3.5) is easy to be obtained:

$$
\sum_{i=1}^{N_{s}} \sum_{j=1}^{N_{s}}\left(n_{k}\right)_{I, j}^{s+1} h^{2}=\sum_{i=1}^{N_{s}} \sum_{i=1}^{N_{s}}\left(n_{k}\right)_{i, j}^{s} h^{2}, \quad k=1,2
$$




\section{Numerical experiments}

In this section, the proposed SAV-CN scheme is used to check the feasibility. The numerical experiments are designed in 2D space with the mixture consists of methane $\left(\mathrm{CH}_{4}\right)$ and $n$-decane $\left(n \mathrm{C}_{10} \mathrm{H}_{22}\right)$. In the following numerical example, the temperature we choose is $450 \mathrm{~K}$. The computational domain is set as a square area $\Omega=\left(0, L_{D}\right)^{2}$ with $L_{D}=2 \times 10^{-8}$ meters and $200 \times 200$ mesh grids are used. The time step $\Delta t=10^{-4}$. The initial values of methane and $n$-decane are shown in Table 1 . The initial condition is to impose the liquid phase of the substances under the saturated steam pressure at $450 \mathrm{~K}$ in the region of $\left(0.3 L_{D}, 0.7 L_{D}\right)^{2}$. The rest of the domain is filled with the mixture of the gas phase of the substances under the same external conditions.

\subsection{Distributions of the molar density and other properties}

Obviously, at the initial state, there exists a jump in molar density between two different phases. During the simulation, the square corners will be changed into round circular corners at the equilibrium state by the surface force. Figuratively, the molar density distributions (Figs. 4 and 5) evolution of methane and $n$-decane match well with those from the component-wise convex splitting scheme in [6].

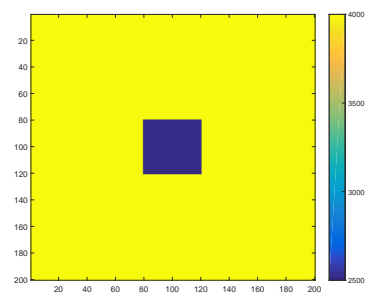

(a)

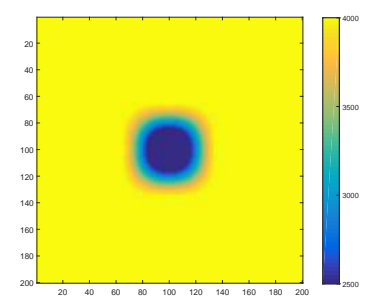

(b)

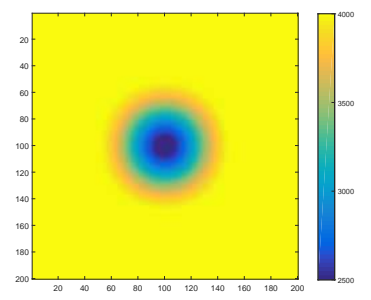

(c)

Figure 4: Molar density distributions of methane at different time: (a) $t=0$, (b) $t=500$, (c) $t=1500$.

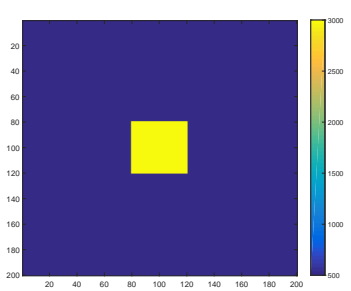

(a)

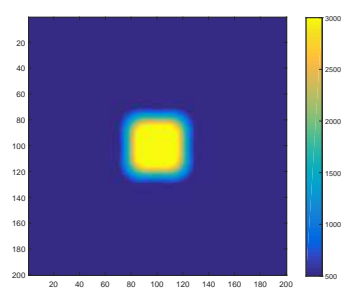

(b)

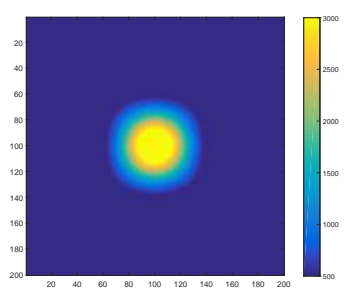

(c)

Figure 5: Molar density distributions of $n$-decane at different time: (a) $t=0$, (b) $t=500$, (c) $t=1500$. 

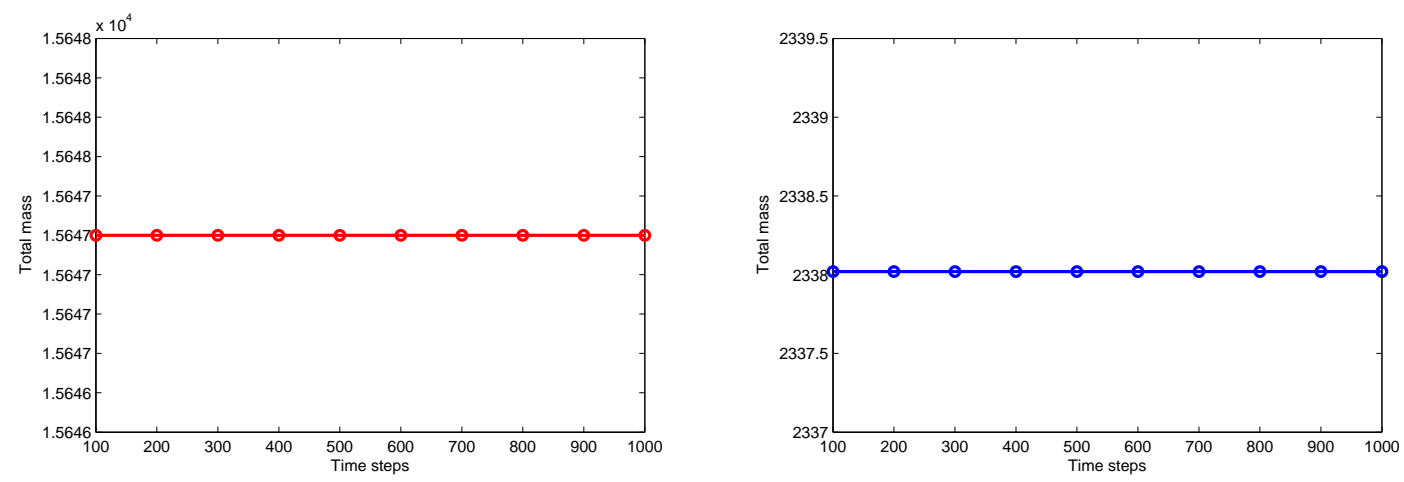

Figure 6: The mass evolution of methane (left) and n-decane (right).

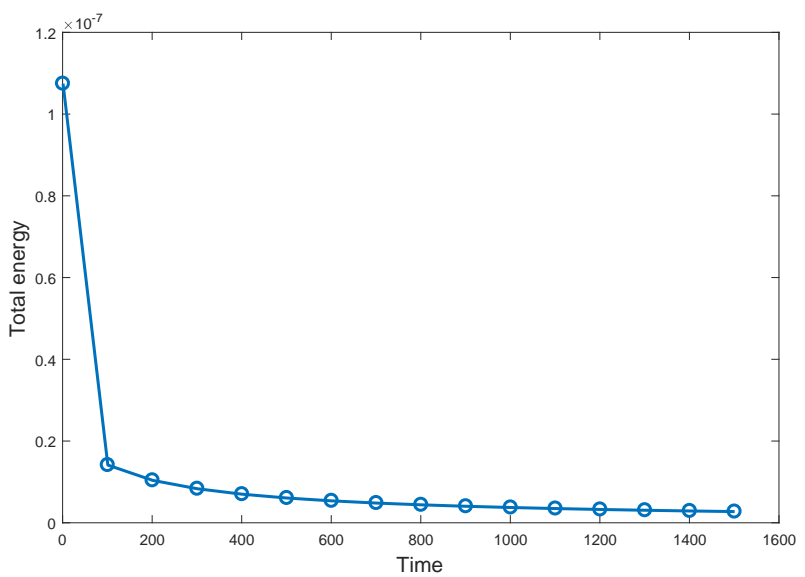

Figure 7: The energy evolution of the system.

The mass evolution of each phase in the process are illustrated in Fig. 6, which verify the mass conservation property of our scheme. Fig. 7 shows the evolution history of the total energy. An obvious energy dissipation trend can be observed.

\subsection{Calculation of the interface tension}

The interface tension $(\mathrm{N} / \mathrm{m})$ is the net contractive force per unit length of the interface which has the following form

$$
\sigma_{\text {sur }}=\frac{F(n)-F\left(n_{\text {initial }}\right)}{A},
$$

where $\mathrm{A}$ is the cross surface area of the liquid drop. In the previous work [22], Qiao and Sun set the radius of the liquid drop under the assumption that the volume of the drop does not change all along the experiment. This assumption comes from the sharp 


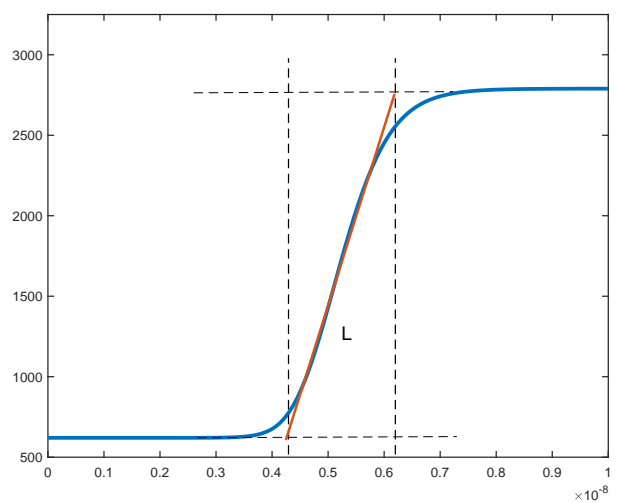

(a)

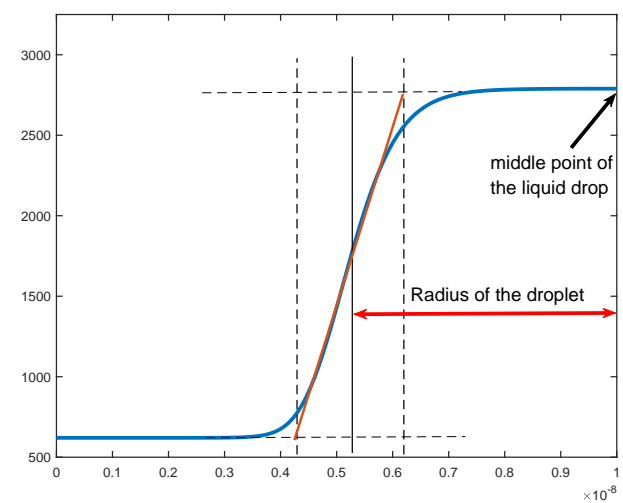

(b)

Figure 8: The width chosen of the interface: (a) Method 1; (b) Method 2.

interface theory. Similarly, the literature $[6,14]$ used the same method to calculate the interface tension. On the other hand, our numerical experiment is based on the diffuse interface theory. When we calculate the area $A$, the thickness of the interface needs to be taken into consideration. Furthermore, in the multi-component case, the diffusion of liquid-gas interface may be different between substances. This could be observed in Figs. 4 and 5. It is nature to determine the calculate area $A$ here as the weighted mean of the area of different substances.

$$
A=\sum_{i} \omega_{i} A_{i}
$$

where $\omega_{i}=\frac{N_{i}}{\sum_{i} N_{i}}$ and $A_{i}$ represents the area of the $i$-th component. Here, we have two different approaches to get the area $A_{i}$.

Method 1. If we considered the width of the interface (which is proposed in [23]), as shown in Fig. 8(a) (here we use the methane as an example). Using the interface width $L$, we can get the radius of the droplet at the stable state as $r=\frac{1}{2} L+r_{0}$ (assuming that the area of the square droplet at the initial state is $A_{s}, r_{0}=\sqrt{\frac{2 A_{s}}{\pi}}$ ). In this approach, we implicitly assumed that the volume (area in 2D) of the liquid droplet is conserved.

Method 2. In general, the volume (area in 2D) of the liquid droplet may not be conserved exactly during the process of the interface formation. Without having to assume the conservation of the liquid droplet volume, we provide another approach to get the radius of the droplet which can be found in Fig. 8(b). Here, we set the distance from the middle point of the interface to the middle point of the pure liquid droplet as the radius to get the area $A_{i}$.

Fig. 9 shows interface tensions calculated by these two approaches. At the same time, the laboratory data and previous results in [6] are also marked. We can see that interface 


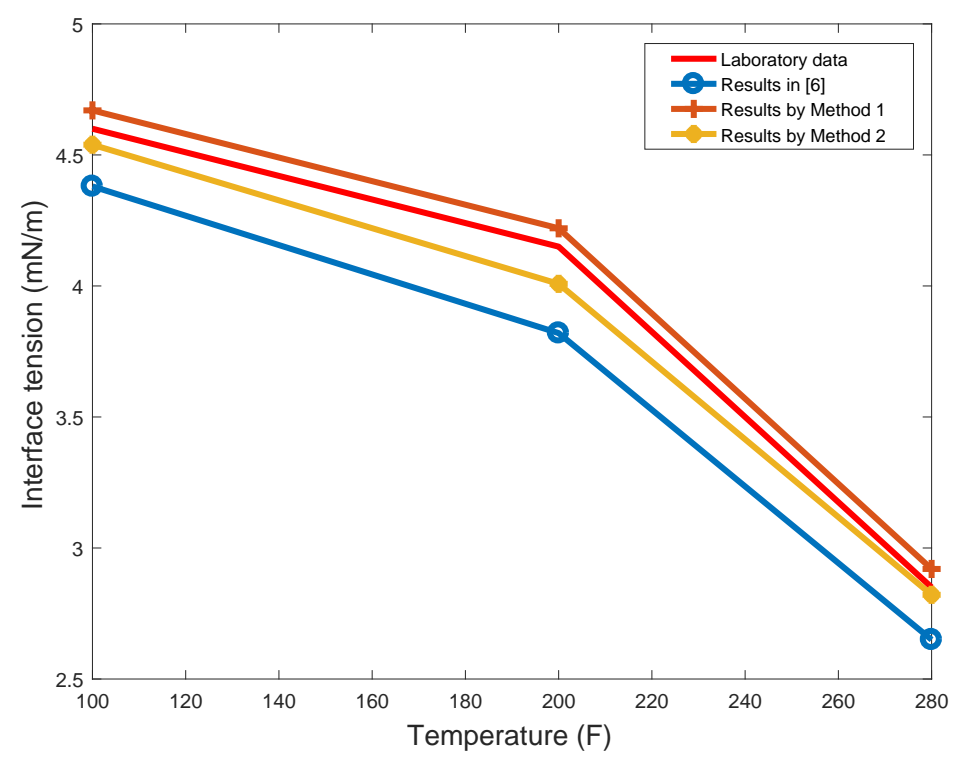

Figure 9: Comparison of interface tensions between the laboratory data and numerical results.

tensions obtained by our methods are much better than those in [6] due to the involving interface width. It is now acceptable from the engineering point of view. We also find that the difference between the two approaches of getting the interface area is relatively small. This can be attributed to the fact that the density of liquid phase is usually much larger than that of the gas phase, especially when hydrocarbon is involved in the substances. When these two phases contribute same mass to the interface, the gas phase will lose much more volume than the liquid phase. Generally, this reflects to the fact that the volume of the liquid phase experiences a small change during the interface formation.

\section{Conclusions}

In this paper, we propose a new multi-component diffuse interface model with the PengRobinson EOS, which is physically consistent. To complete the system, we introduce a method to calculate the initial values based on the NVT flash calculation. The SAV approach is employed to develop efficient numerical schemes for solving the investigated model. Energy stability is derived in the sense that the modified discrete energy is nonincreasing in time. Numerical experiments are conducted with realistic hydrocarbons methane $\left(\mathrm{CH}_{4}\right)$ and $n$-decane $\left(n \mathrm{C}_{10} \mathrm{H}_{22}\right)$. Two different methods are given to calculate the interface tension. Our numerical results agree better with the laboratory experimental data compared with existing results in the literature. 


\section{Acknowledgments}

Z. Qiao's work is partially supported by Hong Kong Research Council GRF grant No. 15325816. S. Sun and T. Zhang gratefully acknowledge that the research reported in this publication was supported by King Abdullah University of Science and Technology (KAUST) through the grant BAS/1/1351-01 and National Nature Science Foundation of China (No. 51874262).

\section{Appendix}

When considering the real inhomogeneous fluid system, the diffuse interface between two phases should be considered. In order to describe the phenomenon around the interface, the diffuse interface model with the gradient contribution is often used. Qiao and Sun [22] proposed a diffuse interface model which is based on Peng-Robinson EOS. In the theory, the Helmholtz free energy is used as the kernel. For describing the behavior on the interfaces of the $m$ components system, we set the system temperature is $T(K)$ and let molar density have the form $\mathbf{n}=\left(n_{1}, n_{2}, \cdots, n_{m}\right)^{T}$, where $n_{i}$ is the molar density of the $i$-th component. In the model, we not only use the homogeneous Helmholtz energy density $f_{b}$ but also need to add a gradient term $f_{\nabla}(\mathbf{n})$ to model the interface effect.

The Helmholtz free energy $f_{b}(\mathbf{n})$ of a homogeneous fluid with Peng-Robinson EOS is given by

$$
\begin{aligned}
& f_{b}(\mathbf{n})=f_{b}^{\text {ideal }}(\mathbf{n})+f_{b}^{\text {excess }}(\mathbf{n}), \\
& f_{b}^{\text {ideal }}(\mathbf{n})=R T \sum_{i=1}^{m} n_{i}\left(\ln n_{i}-1\right), \\
& f_{b}^{\text {excess }}(\mathbf{n})=-n R T \ln (1-b n)+\frac{a(T) n}{2 \sqrt{2} b} \ln \left(\frac{1+(1-\sqrt{2}) b n}{1+(1+\sqrt{2}) b n}\right),
\end{aligned}
$$

where $n=\sum_{i}^{m} n_{i}$ is the total molar density of the system, $R$ is the gas constant which has the value $8.31432 \mathrm{JK}^{-1} \mathrm{~mol}^{-1} . a=a(T)$ is the pressure correction coefficient and $b=b(T)$ is the volume correction coefficient, which have the following form

$$
a(T)=\sum_{i=1}^{m} \sum_{j=1}^{m} y_{i} y_{j}\left(a_{i} a_{j}\right)^{\frac{1}{2}}\left(1-k_{i j}\right), \quad b(T)=\sum_{i=1}^{m} y_{i} b_{i} .
$$

Here $y_{i}$ represents the mole fraction of the $i$-th component, $k_{i j}$ represents the binary interaction of Peng-Robinson EOS. $a_{i}$ and $b_{i}$ in the equation above are shown as follows

$$
\begin{aligned}
& a_{i}(T)=0.45724 \frac{R^{2} T_{c i}^{2}}{P_{c i}}\left(1+m_{i}\left(1-\sqrt{\frac{T}{T_{c i}}}\right)\right)^{2} ; \\
& b_{i}=0.7780 \frac{R T_{c i}}{P_{c i}},
\end{aligned}
$$


where $T_{c i}$ and $P_{c i}$ are properties of the $i$-th substance which represent the critical temperature and critical pressure, respectively. $m_{i}$ has the form

$$
\begin{aligned}
& m_{i}=0.37464+1.54226 \omega_{i}-0.26992 \omega_{i}^{2}, \quad \omega_{i} \leq 0.49 ; \\
& m_{i}=0.379642+1.485030 \omega_{i}-0.164423 \omega_{i}^{2}+0.01666666 \omega_{i}^{3} ; \quad \omega_{i}>0.49,
\end{aligned}
$$

where $\omega_{i}$ can be achieved by using the critical data of the substances as follows

$$
\omega_{i}=\frac{3}{7}\left(\frac{\log _{10}\left(\frac{P_{c i}}{14.695 P S I}\right)}{\frac{T_{c i}}{T_{b i}}-1}\right)-1 .
$$

The inhomogeneous term of the gradient contribution $f_{\nabla}(n)$ can be modeled by a simple relation:

$$
f_{\nabla}(\mathbf{n})=\frac{1}{2} \sum_{i, j=1}^{m} c_{i j} \nabla n_{i} \cdot \nabla n_{j}
$$

where $c_{i j}$ is the influence parameter

$$
c_{i j}=\left(1-\beta_{i j}\right) \sqrt{c_{i} c_{j}} .
$$

Here, $\beta_{i j}$ is the binary coefficient with $\beta_{i i}=0$. And we set $\beta_{i j}=0.5$ as in [13] when $i \neq$ $j$. $c_{i}$ represents the influence of the pure substance. It has relations with the pressure correction parameter and the volume correction parameter of Peng-Robinson EOS.

$$
\begin{aligned}
& c_{i}=a_{i} b_{i}^{\frac{3}{2}}\left(m_{1, i}^{c}\left(1-\frac{T_{c i}}{T}+m_{2, i}^{c}\right)\right), \\
& m_{1, i}^{c}=\frac{10^{-16}}{1.2326+1.357457 \omega_{i}}, \\
& m_{2, i}^{c}=\frac{10^{-16}}{0.9051+1.5410 \omega_{i}} .
\end{aligned}
$$

After adding the gradient contribution $f_{\nabla}$, the total Helmholtz free energy is presented as

$$
f(\mathbf{n})=f_{b}(\mathbf{n})+f_{\nabla}(\mathbf{n}) .
$$

\section{References}

[1] A. Baskaran, J. S. Lowengrub, C. Wang, and S. M. Wise. Convergence analysis of a second order convex splitting scheme for the modified phase field crystal equation. SIAM Journal on Numerical Analysis, 51(5):2851-2873 0036-1429, 2013.

[2] Z. Chen, G. Huan, and Y. Ma. Computational methods for multi-phase flows in porous media, volume 2 Siam, 2006.

[3] D. A. Cogswell. A phase-field study of ternary multi-phase microstructures, 2010. 
[4] H. Dong, Z. Qiao, S. Sun, and T. Tang. Adaptive moving grid methods for two-phase flow in porous media. Journal of Computational and Applied Mathematics, 265:139-150, 2014.

[5] C. M. Elliott and A. Stuart. The global dynamics of discrete semi-linear parabolic equations. SIAM Journal on Numerical Analysis, 30(6):1622-1663 0036-1429, 1993.

[6] X. Fan, J. Kou, Z. Qiao, and S. Sun. A componentwise convex splitting scheme for diffuse interface models with van der waals and Peng-Robinson equations of state. SIAM Journal on Scientific Computing, 39(1):B1-B28, 2017.

[7] T. Jindrová and J. Mikyška. Fast and robust algorithm for calculation of two-phase equilibria at given volume, temperature, and moles. Fluid Phase Equilibria, 353:101-114, 2013.

[8] T. Jindrová and J. Mikyška. General algorithm for multiphase equilibria calculation at given volume, temperature, and moles. Fluid Phase Equilibria, 393:7-25, 2015.

[9] J. Kou and S. Sun. Numerical methods for a multi-component two-phase interface model with geometric mean influence parameters. SIAM Journal on Scientific Computing, 37(4):B543B569 1064-8275, 2015.

[10] J. Kou and S. Sun. Unconditionally stable methods for simulating multi-component twophase interface models with Peng-Robinson equation of state and various boundary conditions. Journal of Computational and Applied Mathematics, 291:158-182 0377-0427, 2016.

[11] J. Kou and S. Sun. A stable algorithm for calculating phase equilibria with capillarity at specified moles, volume and temperature using a dynamic model. Fluid Phase Equilibria, 456:7-24, 2018.

[12] J. Kou and S. Sun. Thermodynamically consistent modeling and simulation of multicomponent two-phase flow with partial miscibility. Computer Methods in Applied Mechanics and Engineering, 331:623-649, 2018.

[13] J. Kou, S. Sun and X. Wang. Linearly Decoupled Energy-Stable Numerical Methods for Multicomponent Two-Phase Compressible Flow. SIAM Journal on Numerical Analysis, 56(6):B543-B569 3219-3248, 2018.

[14] H. Li, L. Ju, C. Zhang, and Q. Peng. Unconditionally energy stable linear schemes for the diffuse interface model with Peng-Robinson equation of state. Journal of Scientific Computing, 75(2):993-1015, 2018.

[15] Y. Li, J. Kou, and S. Sun. Numerical modeling of isothermal compositional grading by convex splitting methods. Journal of Natural Gas Science and Engineering, 43:207-221, 2017.

[16] J. Mikyka and A. Firoozabadi. Investigation of mixture stability at given volume, temperature, and number of moles Fluid Phase Equilibria, 321:1-9 2012.

[17] J. Moortgat, S. Sun and A. Firoozabadi. Compositional modeling of three-phase flow with gravity using higher-order finite element methods. Water Resources Research, 47(5), 2011.

[18] D. Y. Peng and D. B. Robinson. A new two-constant equation of state. Industrial \& Engineering Chemistry Fundamentals, 15(1):59-64, 1976.

[19] Q. Peng. A convex-splitting scheme for a diffuse interface model with Peng-Robinson equation of state. Advances in Applied Mathematics and Mechanics, 9(5):1162-1188, 2017.

[20] Q. Peng, Z. Qiao and S. Sun. Stability and convergence analysis of second-order schemes for a diffuse interface model with Peng-Robinson equation of state. Journal of Computational Mathematics, 35: 737-765, 2017.

[21] O. Polívka and J. Mikyška. Compositional modeling in porous media using constant volume flash and flux computation without the need for phase identification. Journal of Computational Physics, 272:149-169, 2014.

[22] Z. Qiao and S. Sun. Two-phase fluid simulation using a diffuse interface model with PengRobinson equation of state. SIAM Journal on Scientific Computing, 36(4):B708-B728, 2014. 
[23] Z. Qiao, X. Yang and Y. Zhang. Mass conservative lattice Boltzmann scheme for a threedimensional diffuse interface model with Peng-Robinson equation of state. Physical Review E, 98(2):023306, 2018.

[24] J. Shen, J. Xu, and J. Yang. A new class of efficient and robust energy stable schemes for gradient flows. arXiv preprint arXiv:1710.01331, 2017

[25] J. Shen, J. Xu, and J. Yang. The scalar auxiliary variable (SAV) approach for gradient flows. Journal of Computational Physics, 353:407-416 2018.

[26] J. Shen and X. Yang. Decoupled, energy stable schemes for phase-field models of two-phase incompressible flows. SIAM Journal on Numerical Analysis, 53(1):279-296 0036-1429, 2015. 